\title{
LIFTING VECTOR FIELDS FROM MANIFOLDS TO THE $r$-JET PROLONGATION OF THE TANGENT BUNDLE
}

\author{
JAN KUREK AND WŁODZIMIERZ M. MIKULSKI
}

\begin{abstract}
If $m \geq 3$ and $r \geq 0$, we deduce that any natural linear operator lifting vector fields from an $m$-manifold $M$ to the $r$-jet prolongation $J^{r} T M$ of the tangent bundle $T M$ is the composition of the flow lifting $\mathcal{J}^{r}$ corresponding to the $r$-jet prolongation functor $J^{r}$ with a natural linear operator lifting vector fields from $M$ to $T M$. If $0 \leq s \leq r$ and $m \geq 3$, we find all natural linear operators transforming vector fields on $M$ into base-preserving fibred maps $J^{r} T M \rightarrow J^{s} T M$.
\end{abstract}

\section{INTRODUCTION}

All manifolds considered in this paper are assumed to be finite dimensional, without boundary, and smooth. Maps between manifolds are assumed to be smooth (of class $C^{\infty}$ ).

The general concept of bundle functors and natural operators can be found in the fundamental monograph [4].

In [1], J. Gancarzewicz proved that any natural linear operator $A$ lifting vector fields $X \in \mathcal{X}(M)$ on an $m$-manifold $M$ into vector fields $A(X) \in \mathcal{X}(T M)$ on the tangent bundle $T M$ of $M$ is of the form $A(X)=a X^{C}+b X^{V}$ for real numbers $a$ and $b$, where $X^{C}=\mathcal{T} X \in \mathcal{X}(T M)$ is the complete (flow) lift of $X$ to $T M$ and $X^{V} \in \mathcal{X}(T M)$ is the vertical lift of $X$ to $T M$.

In this paper, we prove that if $m \geq 3$ then any natural linear operator $A$ lifting vector fields $X \in \mathcal{X}(M)$ on an $m$-manifold $M$ into vector fields $A(X) \in \mathcal{X}\left(J^{r} T M\right)$ on the $r$-jet prolongation $J^{r} T M$ of $T M$ is of the form

$$
A(X)=a \mathcal{J}^{r} X^{C}+b \mathcal{J}^{r} X^{V}
$$

for (uniquely determined) real numbers $a$ and $b$.

Moreover, if $0 \leq s \leq r$ and $m \geq 3$, we find all natural linear operators $A$ transforming vector fields $X \in \mathcal{X}(M)$ on an $m$-manifold $M$ into base-preserving fibred maps $A(X): J^{r} T M \rightarrow J^{s} T M$.

2010 Mathematics Subject Classification. Primary: 58A20, 53A55.

Key words and phrases. Natural bundle; natural operator; vector field; jet prolongation functor; tangent bundle. 
Natural operators lifting functions and vector fields are applied in almost all investigations of prolongation of geometric structures, see e.g. [8, 9]. That is why such natural operators are studied in many papers, see e.g. [1, 2, 3, 4, 15, 6, 7].

From now on, let $x^{1}, \ldots, x^{m}$ denote the usual coordinates on $\mathbf{R}^{m}$ and $\partial_{1}, \ldots, \partial_{m}$ be the canonical vector fields on $\mathbf{R}^{m}$.

\section{Preliminaries}

Let $\mathcal{M} f_{m}$ be the category of $m$-dimensional manifolds and their local diffeomorphisms; let $\mathcal{F} \mathcal{M}$ be the category of fibred manifolds (i.e. surjective submersions between manifolds) and their fibred maps; let $\mathcal{F} \mathcal{M}_{m}$ be the category of fibred manifolds with $m$-dimensional bases and their fibred maps with local diffeomorphisms as base maps; and let $\mathcal{V B}$ be the category of vector bundles and their vector bundle homomorphisms.

The $r$-jet prolongation $J^{r} Y$ of an $\mathcal{F} \mathcal{M}_{m}$-object $Y=(Y \rightarrow M)$ is the space of $r$-jets $j_{x}^{r} \sigma$ at points $x \in M$ of local sections $\sigma$ of $Y$. It is a fibre bundle over $Y$ with projection $j_{x}^{r} \sigma \mapsto \sigma(x)$. Every $\mathcal{F M}_{m}$-map $f: Y \rightarrow Y_{1}$ with the base map $\underline{f}: M \rightarrow M_{1}$ induces the fibred map $J^{r} f: J^{r} Y \rightarrow J^{r} Y_{1}$ by $j_{x}^{r} \sigma \mapsto j_{f(x)}^{r}\left(f \circ \sigma \circ \underline{f}^{-1}\right)$. The resulting functor $J^{r}: \mathcal{F} \mathcal{M}_{m} \rightarrow \mathcal{F M}$ is a bundle functor in the sense of [4].

Let $Y=(Y \rightarrow M)$ be an $\mathcal{F} \mathcal{M}_{m}$-object. A vector field $Z \in \mathcal{X}(Y)$ is called projectable if there is a vector field $\underline{Z} \in \mathcal{X}(M)$ on $M$ being related with $Z$ with respect to the projection $Y \rightarrow M$. We denote by $\mathcal{X}_{\text {proj }}(Y)$ the space of projectable vector fields on $Y$. Equivalently, $Z \in \mathcal{X}(Y)$ is projectable if and only if the flow $\left\{\mathrm{Fl}_{t}^{Z}\right\}$ of $Z$ is formed by $\mathcal{F} \mathcal{M}_{m}$-maps. Thus for any $Z \in \mathcal{X}_{\text {proj }}(Y)$ we have $\mathcal{J}^{r} Z \in$ $\mathcal{X}\left(J^{r} Y\right)$ given by $\mathcal{J}^{r} Z=\left.\frac{\partial}{\partial t}\right|_{t=0} J^{r} \mathrm{Fl}_{t}^{Z}$.

Let $T: \mathcal{M} f_{m} \rightarrow \mathcal{F} \mathcal{M}_{m}$ be the (usual) tangent functor sending any $m$-manifold $M$ into the tangent bundle $T M$ of $M$ and any $\mathcal{M} f_{m}$-map $\varphi: M \rightarrow M_{1}$ into the tangent map $T \varphi: T M \rightarrow T M_{1}$ of $\varphi$. Composing $T$ with $J^{r}$ we obtain the bundle functor $J^{r} T: \mathcal{M} f_{m} \rightarrow \mathcal{F} \mathcal{M}$ sending any $m$-manifold $M$ into the space $J^{r} T M$ of $r$ jets $j_{x}^{r} X$ at points $x \in M$ of vector fields $X$ on $M$ and every $\mathcal{M} f_{m}$-map $\varphi: M \rightarrow N$ of two $m$-manifolds into $J^{r} T \varphi: J^{r} T M \rightarrow J^{r} T N$ given by $J^{r} T \varphi\left(j_{x}^{r} X\right)=j_{\varphi(x)}^{r}(T \varphi \circ$ $X \circ \varphi^{-1}$ ). We see that $J^{r} T M$ is (in the obvious way) a vector bundle over $M$ and $J^{r} T \varphi: J^{r} T M \rightarrow J^{r} T N$ is a vector bundle map. So, $J^{r} T: \mathcal{M} f_{m} \rightarrow \mathcal{V B}$.

\section{NATURAL OPERATORS}

An $\mathcal{M} f_{m}$-natural linear operator $A: T_{\mid \mathcal{M} f_{m}} \rightsquigarrow T\left(J^{r} T\right)$ (lifting vector fields from $m$-manifolds to the $r$-jet prolongation of the tangent bundle) is an $\mathcal{M} f_{m}$-invariant family of $\mathbf{R}$-linear operators (R-linear functions)

$$
A: \mathcal{X}(M) \rightarrow \mathcal{X}\left(J^{r} T M\right)
$$

for all $m$-manifolds $M$, where $\mathcal{X}(M)$ is the vector space of vector fields on $M$. The invariance of $A$ means that if $X \in \mathcal{X}(M)$ and $X_{1} \in \mathcal{X}\left(M_{1}\right)$ are $\varphi$-related (i.e. $T \varphi \circ X=X_{1} \circ \varphi$ ) for a $\mathcal{M} f_{m}$-map $\varphi: M \rightarrow M_{1}$, then $A(X)$ and $A\left(X_{1}\right)$ are $J^{r} T \varphi$-related. 
Example 3.1. Let $X \in \mathcal{X}(M)$ be a vector field on an $m$-manifold $M$. We have the (complete) flow lift $X^{C}=\mathcal{T} X \in \mathcal{X}_{\text {proj }}(T M)$ of $X$ to $T M$. So, we have $\mathcal{J}^{r} X^{C} \in \mathcal{X}\left(J^{r} T M\right)$. Alternatively, $\mathcal{J}^{r} X^{C}$ is the flow lift of $X$ to $J^{r} T M$ via the bundle functor $J^{r} T$. The function $\mathcal{X}(M) \rightarrow \mathcal{X}\left(J^{r} T M\right)$ given by $X \mapsto \mathcal{J}^{r} X^{C}$ is $\mathbf{R}$-linear. The resulting family $T_{\mid \mathcal{M} f_{m}} \rightsquigarrow T\left(J^{r} T\right)$ is an $\mathcal{M} f_{m}$-natural linear operator.

Example 3.2. Let $X \in \mathcal{X}(M)$ be as above. We have the vertical lift $X^{V} \in$ $\mathcal{X}_{\text {proj }}(T M)$ of $X$ to $T M$. So, we have $\mathcal{J}^{r} X^{V} \in \mathcal{X}\left(J^{r} T M\right)$. Clearly, $\mathcal{J}^{r} X_{\mid j_{x}^{r} Y}^{V}=$ $\left.\frac{d}{d t}\right|_{t=0}\left(j_{x}^{r} Y+t j_{x}^{r} X\right)$. The function $\mathcal{X}(M) \rightarrow \mathcal{X}\left(J^{r} T M\right)$ given by $X \mapsto \mathcal{J}^{r} X^{V}$ is $\mathbf{R}$-linear. The resulting family $T_{\mid \mathcal{M} f_{m}} \rightsquigarrow T\left(J^{r} T\right)$ is an $\mathcal{M} f_{m}$-natural linear operator.

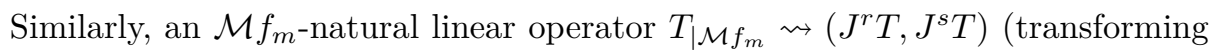
vector fields on $m$-manifolds into fibred base-preserving maps from the $r$-jet prolongation of the tangent bundle into the $s$-jet prolongation of the tangent bundle) is an $\mathcal{M} f_{m}$-invariant family of $\mathbf{R}$-linear operators ( $\mathbf{R}$-linear functions)

$$
A: \mathcal{X}(M) \rightarrow C_{M}^{\infty}\left(J^{r} T M, J^{s} T M\right)
$$

for all $m$-manifolds $M$, where $\mathcal{X}(M)$ is the vector space of vector fields on $M$ and $C_{M}^{\infty}\left(J^{r} T M, J^{s} T M\right)$ is the vector space of base-preserving fibred maps $J^{r} T M \rightarrow$ $J^{s} T M$. The invariance of $A$ means that if $X \in \mathcal{X}(M)$ and $X_{1} \in \mathcal{X}\left(M_{1}\right)$ are $\varphi$-related vector fields for an $\mathcal{M} f_{m}$-map $\varphi: M \rightarrow M_{1}$, then so are $A(X): J^{r} T M \rightarrow$ $J^{s} T M$ and $A\left(X_{1}\right): J^{r} T M_{1} \rightarrow J^{s} T M_{1}$ (i.e. $J^{s} T \varphi \circ A(X)=A\left(X_{1}\right) \circ J^{r} T \varphi$ ).

Example 3.3. Let $k$ be an integer such that $0 \leq k \leq r-s$. Given a vector field $X \in \mathcal{X}(M)$ on an $m$-manifold $M$ we have a base-preserving fibred map

$$
A^{\langle k\rangle}(X): J^{r} T M \rightarrow J^{s} T M, \quad A^{\langle k\rangle}(X)\left(j_{x}^{r} Y\right)=j_{x}^{s}\left(\operatorname{ad}_{Y}^{k}(X)\right),
$$

where $\operatorname{ad}_{Y}: \mathcal{X}(M) \rightarrow \mathcal{X}(M)$ is the adjoint map given by $\operatorname{ad}_{Y}(X)=[Y, X]$ and $\operatorname{ad}_{Y}^{k}=\operatorname{ad}_{Y} \circ \cdots \circ \operatorname{ad}_{Y}(k$ times $)$. Thus we have the resulting $\mathcal{M} f_{m}$-natural linear operator $A^{\langle k\rangle}: T_{\mid \mathcal{M} f_{m}} \rightsquigarrow\left(J^{r} T, J^{s} T\right)$.

\section{Preparatory lemmas}

Lemma 4.1. Let $A: T_{\mid \mathcal{M} f_{m}} \rightsquigarrow\left(J^{r} T, J^{s} T\right)$ be an $\mathcal{M} f_{m}$-natural linear operator with $A\left(\left(x^{1}\right)^{q} \partial_{2}\right)\left(j_{0}^{r} \partial_{1}\right)=0$ for $q=0, \ldots, r$. If $0 \leq s \leq r$ and $m \geq 2$, then $A=0$.

Proof. First, prove that

$$
A\left(x^{\alpha} \partial_{j}\right)\left(j_{0}^{r} \partial_{1}\right)=0
$$

for any $\alpha \in(\mathbf{N} \cup\{0\})^{m}$ and any $j=1, \ldots, m$. Let us consider three cases.

(I) Let $\alpha=\left(\alpha_{1}, \ldots, \alpha_{m}\right) \in(\mathbf{N} \cup\{0\})^{m}$ be such that $|\alpha| \leq r$ and let $j \in$ $\{2, \ldots, m\}$. By the Frobenius theorem there exists a local embedding $\varphi: \mathbf{R}^{m} \rightarrow$ $\mathbf{R}^{m}$ of the form $\operatorname{id}_{\mathbf{R}} \times \psi$ such that $\varphi_{*} \partial_{2}=\partial_{2}+\left(x^{2}\right)^{\alpha_{2}} \ldots\left(x^{m}\right)^{\alpha_{m}} \partial_{j}$ on some neighborhood of 0 . Then $\varphi_{*} \partial_{1}=\partial_{1}$ and $\varphi_{*}\left(\left(x^{1}\right)^{\alpha_{1}} \partial_{2}\right)=\left(x^{1}\right)^{\alpha_{1}} \partial_{2}+x^{\alpha} \partial_{j}$ in some 
neighborhood of 0 . On the other hand, since $\alpha_{1} \leq r$, by the assumption of the lemma we have

$$
A\left(\left(x^{1}\right)^{\alpha_{1}} \partial_{2}\right)\left(j_{0}^{r} \partial_{1}\right)=0 .
$$

Then, using the invariance of $A$ with respect to $\varphi$, we obtain

$$
A\left(\left(x^{1}\right)^{\alpha_{1}} \partial_{2}+x^{\alpha} \partial_{j}\right)\left(j_{0}^{r} \partial_{1}\right)=0 .
$$

Hence, we have (4.1) for any $\alpha=\left(\alpha_{1}, \ldots, \alpha_{m}\right) \in(\mathbf{N} \cup\{0\})^{m}$ with $|\alpha| \leq r$ and any $j \in\{2, \ldots, m\}$.

(II) Let $\alpha=\left(\alpha_{1}, \ldots, \alpha_{m}\right) \in(\mathbf{N} \cup\{0\})^{m}$ be such that $|\alpha| \leq r$ and let $j=1$. For any $\tau \in \mathbf{R}$, the linear isomorphism $\left(x^{1}+\tau x^{2}, x^{2}, \ldots, x^{m}\right)$ preserves $\partial_{1}$ and sends $x^{\alpha} \partial_{2}$ into $\left(x^{1}-\tau x^{2}\right)^{\alpha_{1}}\left(x^{2}\right)^{\alpha_{2}} \ldots\left(x^{m}\right)^{\alpha_{m}}\left(\partial_{2}+\tau \partial_{1}\right)$. Further, from the case (I) we have $A\left(x^{\alpha} \partial_{2}\right)\left(j_{0}^{r} \partial_{1}\right)=0$. So, using the invariance of $A$ with respect to $\left(x^{1}+\right.$ $\left.\tau x^{2}, x^{2}, \ldots, x^{m}\right)$, we obtain

$$
A\left(\left(x^{1}-\tau x^{2}\right)^{\alpha_{1}}\left(x^{2}\right)^{\alpha_{2}} \ldots\left(x^{m}\right)^{\alpha_{m}}\left(\partial_{2}+\tau \partial_{1}\right)\right)\left(j_{0}^{r} \partial_{1}\right)=0 .
$$

Both sides of the last equality are polynomials in $\tau$. Considering the coefficients of the polynomials on $\tau$, we obtain

$$
A\left(x^{\alpha} \partial_{1}\right)\left(j_{0}^{r} \partial_{1}\right)-\alpha_{1} A\left(\left(x^{1}\right)^{\alpha_{1}-1}\left(x^{2}\right)^{\alpha_{2}+1} \ldots\left(x^{m}\right)^{\alpha_{m}} \partial_{2}\right)\left(j_{0}^{r} \partial_{1}\right)=0 .
$$

(If $\alpha_{1}=0$ the term $\alpha_{1} A(\ldots)\left(j_{0}^{r} \partial_{1}\right)$ does not occur.) Further, from the case (I) we have $\alpha_{1} A\left(\left(x^{1}\right)^{\alpha_{1}-1}\left(x^{2}\right)^{\alpha_{2}+1} \ldots\left(x^{m}\right)^{\alpha_{m}} \partial_{2}\right)\left(j_{0}^{r} \partial_{1}\right)=0$. Hence we have 4.1 for any $\alpha \in(\mathbf{N} \cup\{0\})^{m}$ with $|\alpha| \leq r$ and $j=1$.

(III) Now, let $\alpha \in(\mathbf{N} \cup\{0\})^{m}$ be such that $|\alpha| \geq r+1$ and $j=1, \ldots, m$. Then $j_{0}^{r}\left(\partial_{2}+x^{\alpha} \partial_{j}\right)=j_{0}^{r} \partial_{2}$. So, by Lemma 42.4 in [4, there exists a local diffeomorphism $\varphi: \mathbf{R}^{m} \rightarrow \mathbf{R}^{m}$ such that $j_{0}^{r+1} \varphi=j_{0}^{r+1}$ id and $\varphi_{*} \partial_{2}=\partial_{2}+x^{\alpha} \partial_{j}$ on some neighborhood of 0 . Clearly, $\varphi$ preserves $j_{0}^{r} \partial_{1}$. Further, from the case (I) for $j=2$ and $\alpha=(0, \ldots, 0)$, we have $A\left(\partial_{2}\right)\left(j_{0}^{r} \partial_{1}\right)=0$. Then by the invariance of $A$ with respect to $\varphi$ we obtain $A\left(\partial_{2}\right)\left(j_{0}^{r} \partial_{1}\right)=A\left(\partial_{2}+x^{\alpha} \partial_{j}\right)\left(j_{0}^{r} \partial_{1}\right)$. Then we have (4.1) for any $\alpha \in(\mathbf{N} \cup\{0\})^{m}$ such that $|\alpha| \geq r+1$ and $j=1, \ldots, m$.

We are now in a position to complete the proof. From the cases (I)-(III) we get (4.1) for any $\alpha \in(\mathbf{N} \cup\{0\})^{m}$ and any $j=1, \ldots, m$. Then from the linearity of $A$ and the Peetre theorem it follows that $A(X)\left(j_{0}^{r} \partial_{1}\right)=0$ for any $X \in \mathcal{X}\left(\mathbf{R}^{m}\right)$. Now, since the $\mathcal{M} f_{m}$-orbit of $j_{0}^{r} \partial_{1}$ is dense in $J^{r} T M$ and $A$ is $\mathcal{M} f_{m}$-invariant, we get that $A(X)=0$ for any $X \in \mathcal{X}(M)$, i.e. $A=0$.

Lemma 4.2. Let $0 \leq s \leq r$ and $m \geq 2$. Let $A: T_{\mid \mathcal{M} f_{m}} \rightsquigarrow\left(J^{r} T, J^{s} T\right)$ be an $\mathcal{M} f_{m}$-natural linear operator. Given $k=0, \ldots, r$ we have

$$
A\left(\left(x^{1}\right)^{k} \partial_{2}\right)\left(j_{0}^{r} \partial_{1}\right)=\sum_{l=0}^{\min (k, s)} \mu_{l}^{k} j_{0}^{s}\left(\left(x^{1}\right)^{l} \partial_{2}\right)
$$

for some (uniquely determined) real numbers $\mu_{l}^{k}$ for $k=0, \ldots, r$ and $l=0, \ldots$, $\min (k, s)$. 
Proof. We can write

$$
A\left(a\left(x^{1}\right)^{k} \partial_{2}\right)\left(b j_{0}^{r} \partial_{1}\right)=\sum_{j=1}^{m} \sum_{|\alpha| \leq s} \lambda_{\alpha}^{j, k}(a, b) j_{0}^{s}\left(x^{\alpha} \partial_{j}\right),
$$

where $\lambda_{\alpha}^{j, k}$ are some (uniquely determined) smooth maps. Using the invariance of $A$ with respect to $\left(\tau_{1} x^{1}, \ldots, \tau_{m} x^{m}\right)$ for $\tau_{1}=1, \tau_{2} \neq 0, \ldots, \tau_{m} \neq 0$, we get the homogeneity condition

$$
\tau_{2} \lambda_{\alpha}^{j, k}(a, b)=\frac{\tau_{j}}{\tau^{\alpha}} \lambda_{\alpha}^{j, k}(a, b) .
$$

Then $\lambda_{\alpha}^{j, k}(a, b)=0$ if $\tau_{2} \neq \frac{\tau_{j}}{\tau^{\alpha}}$. Hence

$$
A\left(a\left(x^{1}\right)^{k} \partial_{2}\right)\left(b j_{0}^{r} \partial_{1}\right)=\sum_{l=0}^{s} \mu_{l}^{k}(a, b) j_{0}^{s}\left(\left(x^{1}\right)^{l} \partial_{2}\right),
$$

where $\mu_{l}^{k}$ are (uniquely determined) smooth maps. Now, using the invariance of $A$ with respect to $\left(\tau x^{1}, x^{2}, \ldots, x^{m}\right)$ for $\tau \neq 0$, we obtain the homogeneity condition

$$
\frac{1}{\tau^{k}} \mu_{l}^{k}(a, \tau b)=\frac{1}{\tau^{l}} \mu_{l}^{k}(a, b)
$$

Consequently, $\mu_{l}^{k}(a, b)=0$ if $l>k$. The proof of the lemma is complete.

Lemma 4.3. Let $0 \leq s \leq r$ and $m \geq 3$. The vector space of all $\mathcal{M} f_{m}$-natural linear operators $A: T_{\mid \mathcal{M} f_{m}} \rightsquigarrow\left(J^{r} T, J^{s} T\right)$ has dimension $\leq r-s+1$.

Proof. Let $A: T_{\mid \mathcal{M} f_{m}} \rightsquigarrow\left(J^{r} T, J^{s} T\right)$ be an $\mathcal{M} f_{m}$-natural linear operator. Let $\mu_{l}^{k}$ for $k=0, \ldots, r$ and $l=0, \ldots, \min (k, s)$ be the real numbers from Lemma 4.2. By Lemma 4.1. $A$ is uniquely determined by this system $\left(\mu_{l}^{k}\right)$ of real numbers. So, it remains to show that the system $\left(\mu_{l}^{k}\right)$ is uniquely determined by the subsystem $\left(\mu_{0}^{k}\right)$ of real numbers $\mu_{0}^{k}$ for $k=0, \ldots, r-s$. Let us consider two cases.

(I) $s=0$. Then $\left(\mu_{l}^{k}\right)=\left(\mu_{0}^{k}\right)$. So, this case is trivial.

(II) $s \geq 1$. We have $\mu_{l}^{k}=\mu_{0}^{0}$ for $k=0$ and $l=0, \ldots, \min (k, s)=0$. So, we can assume $k \geq 1$. For a real number $\tau$, let $\psi_{\tau}: \mathbf{R}^{m-1} \rightarrow \mathbf{R}^{m-1}$ be a local diffeomorphism such that $\left(\psi_{\tau}\right)_{*} \partial_{2}=\partial_{2}+\tau x^{2} \partial_{2}$ on some neighborhood of 0 . Then from the invariance of $A$ with respect to $\operatorname{id}_{\mathbf{R}} \times \psi_{\tau}$ and 4.2 for $k-1$ instead of $k$ it follows that

$$
A\left(\left(x^{1}\right)^{k-1}\left(\partial_{2}+\tau x^{2} \partial_{2}\right)\right)\left(j_{0}^{r} \partial_{1}\right)=\sum_{l=0}^{\min (k-1, s)} \mu_{l}^{k-1} j_{0}^{s}\left(\left(x^{1}\right)^{l}\left(\partial_{2}+\tau x^{2} \partial_{2}\right)\right) .
$$

Consequently, if we consider the coefficients on $\tau$ of both sides, we get

$$
A\left(\left(x^{1}\right)^{k-1} x^{2} \partial_{2}\right)\left(j_{0}^{r} \partial_{1}\right)=\sum_{l=0}^{\min (k-1, s)} \mu_{l}^{k-1} j_{0}^{s}\left(\left(x^{1}\right)^{l} x^{2} \partial_{2}\right)
$$


Similarly, from the invariance of $A$ with respect to $\left(x^{1}+\tau x^{2}, x^{2}, \ldots, x^{m}\right)$ and 4.2 it follows that

$$
A\left(\left(x^{1}-\tau x^{2}\right)^{k}\left(\partial_{2}+\tau \partial_{1}\right)\right)\left(j_{0}^{r} \partial_{1}\right)=\sum_{l=0}^{\min (k, s)} \mu_{l}^{k} j_{0}^{s}\left(\left(x^{1}-\tau x^{2}\right)^{l}\left(\partial_{2}+\tau \partial_{1}\right)\right) .
$$

So, we have

$$
\begin{aligned}
& -k A\left(\left(x^{1}\right)^{k-1} x^{2} \partial_{2}\right)\left(j_{0}^{r} \partial_{1}\right)+A\left(\left(x^{1}\right)^{k} \partial_{1}\right)\left(j_{0}^{r} \partial_{1}\right) \\
& =-\sum_{l=0}^{\min (k, s)} l \mu_{l}^{k} j_{0}^{s}\left(\left(x^{1}\right)^{l-1} x^{2} \partial_{2}\right)+\sum_{l=0}^{\min (k, s)} \mu_{l}^{k} j_{0}^{s}\left(\left(x^{1}\right)^{l} \partial_{1}\right) .
\end{aligned}
$$

From 4.3 and 4.4 we get

$$
\begin{aligned}
& A\left(\left(x^{1}\right)^{k} \partial_{1}\right)\left(j_{0}^{r} \partial_{1}\right)=k \sum_{l=0}^{\min (k-1, s)} \mu_{l}^{k-1} j_{0}^{s}\left(\left(x^{1}\right)^{l} x^{2} \partial_{2}\right) \\
& -\sum_{l=0}^{\min (k, s)} l \mu_{l}^{k} j_{0}^{s}\left(\left(x^{1}\right)^{l-1} x^{2} \partial_{2}\right)+\sum_{l=0}^{\min (k, s)} \mu_{l}^{k} j_{0}^{s}\left(\left(x^{1}\right)^{l} \partial_{1}\right) .
\end{aligned}
$$

(If $l=s$ then $j_{0}^{s}\left(\left(x^{1}\right)^{l} x^{2} \partial_{2}\right)=0$. If $l=0$, then $l \mu_{l}^{k} j_{0}^{s}\left(\left(x^{1}\right)^{l-1} x^{2} \partial_{2}\right)$ does not occur.) Using the invariance of $A$ with respect to the embedding switching $x^{2}$ and $x^{3}$ (we use the assumption $m \geq 3$ ) and preserving the other coordinates, from 4.5 we get

$$
\begin{aligned}
& A\left(\left(x^{1}\right)^{k} \partial_{1}\right)\left(j_{0}^{r} \partial_{1}\right)=k \sum_{l=0}^{\min (k-1, s)} \mu_{l}^{k-1} j_{0}^{s}\left(\left(x^{1}\right)^{l} x^{3} \partial_{3}\right) \\
& -\sum_{l=0}^{\min (k, s)} l \mu_{l}^{k} j_{0}^{s}\left(\left(x^{1}\right)^{l-1} x^{3} \partial_{3}\right)+\sum_{l=0}^{\min (k, s)} \mu_{l}^{k} j_{0}^{s}\left(\left(x^{1}\right)^{l} \partial_{1}\right) .
\end{aligned}
$$

By (4.5) and (4.6), we see that the coefficients on $j_{0}^{s}\left(\left(x^{1}\right)^{l-1} x^{2} \partial_{2}\right)$ (on the right hand side of (4.5) must be 0 , i.e.

$$
-l \mu_{l}^{k}+k \mu_{l-1}^{k-1}=0
$$

for $l=1, \ldots, \min (k, s)$. So, by induction, the system $\left(\mu_{l}^{k}\right)$ is uniquely determined by $\mu_{0}^{0}, \ldots, \mu_{0}^{r-s}$. The proof of the lemma is complete.

Lemma 4.4. Let $0 \leq s \leq r$ and $m \geq 1$. The system of $\mathcal{M} f_{m}$-natural linear operators $A^{\langle k\rangle}$ from Example 3.3 for $k=0, \ldots, r-s$ is linearly independent.

Proof. Suppose $\sum_{k=0}^{r-s} \lambda_{k} A^{\langle k\rangle}=0$. We prove that $\lambda_{0}=\cdots=\lambda_{q}=0$ for $q=$ $0, \ldots, r-s$. We proceed by induction with respect to $q$.

(i) We start with $q=0$. Since $A^{\langle 0\rangle}\left(\partial_{1}\right)\left(j_{0}^{r} \partial_{1}\right)=j_{0}^{s} \partial_{1}$ and $A^{\langle k\rangle}\left(\partial_{1}\right)\left(j_{0}^{r} \partial_{1}\right)=0$ for $k=1, \ldots, r-s$, then $0=\sum_{k=0}^{r-s} \lambda_{k} A^{\langle k\rangle}\left(\partial_{1}\right)\left(j_{0}^{r} \partial_{1}\right)=\lambda_{0} j_{0}^{s} \partial_{1}$. Then $\lambda_{0}=0$.

(ii) Now, we make the inductive step. Let $r-s-1 \geq q \geq 0$ and assume that $\lambda_{0}=\cdots=\lambda_{q}=0$. Then $0=\sum_{k=0}^{r-s} \lambda_{k} A^{\langle k\rangle}\left(\frac{1}{(q+1) !}\left(x^{1}\right)^{q+1} \partial_{1}\right)\left(j_{0}^{r} \partial_{1}\right)=\lambda_{q+1} j_{0}^{s} \partial_{1}$, 
because $A^{\langle q+1\rangle}\left(\frac{1}{(q+1) !}\left(x^{1}\right)^{q+1} \partial_{1}\right)\left(j_{0}^{r} \partial_{1}\right)=j_{0}^{s} \partial_{1}$ and $A^{\langle k\rangle}\left(\left(x^{1}\right)^{q+1} \partial_{1}\right)\left(j_{0}^{r} \partial_{1}\right)=0$ for $k=q+2, \ldots, r-s$. Then $\lambda_{q+1}=0$, i.e. $\lambda_{0}=\cdots=\lambda_{q+1}=0$, as well.

Thus we have proved that $\lambda_{0}=\cdots=\lambda_{q}=0$ for $q=0, \ldots, r-s$. For $q=r-s$ we get $\lambda_{0}=\cdots=\lambda_{r-s}=0$. The proof of the lemma is complete.

\section{MAIN RESUlts}

Theorem 5.1. Let $0 \leq s \leq r$ and $m \geq 3$. Any $\mathcal{M} f_{m}$-natural linear operator $A: T_{\mid \mathcal{M} f_{m}} \rightsquigarrow\left(J^{r} T, J^{s} T\right)$ is the linear combination of $A^{\langle k\rangle}$ for $k=0, \ldots, r-s$ with (uniquely determined) real coefficients.

Proof. It is an immediate consequence of Lemmas 4.3 and 4.4 .

Theorem 5.2. Let $m \geq 3$ and $r \geq 0$ be integers. Any $\mathcal{M} f_{m}$-natural linear operator $A: T_{\mid \mathcal{M} f_{m}} \rightsquigarrow T\left(J^{r} T\right)$ is of the form (1.1) for (uniquely determined) reals a and $b$.

Proof. Let $A: T_{\mid \mathcal{M} f_{m}} \rightsquigarrow T\left(J^{r} T\right)$ be an $\mathcal{M} f_{m}$-natural linear operator.

Using the source projection $\pi^{r}: J^{r} T M \rightarrow M$ we produce the $\mathcal{M} f_{m}$-natural linear operator $T \pi^{r} \circ A: T_{\mid \mathcal{M} f_{m}} \rightsquigarrow\left(J^{r} T, J^{0} T\right)$. By Theorem 5.1 for $s=0$,

$$
T \pi^{r} \circ A=\sum_{k=0}^{r} \lambda_{k} A^{\langle k\rangle}
$$

where $\lambda_{k}$ are the real numbers. First, we are going to prove that $\lambda_{1}=\cdots=\lambda_{r}=0$.

It is easy to see that $A^{\langle k\rangle}\left(\frac{1}{q !}\left(x^{1}\right)^{q} \partial_{1}\right)\left(j_{0}^{r} \partial_{1}\right)=\delta_{k, q} \partial_{1 \mid 0}$ (the Kronecker delta). So, $T \pi^{r} \circ A\left(\frac{1}{k !}\left(x^{1}\right)^{k} \partial_{1}\right)\left(j_{0}^{r} \partial_{1}\right)=\lambda_{k} \partial_{1 \mid 0}$. Then

$$
A\left(\frac{1}{k !}\left(x^{1}\right)^{k} \partial_{1}\right)\left(j_{0}^{r} \partial_{1}\right)=\lambda_{k} \mathcal{J}^{r} \partial_{1}^{C}\left(j_{0}^{r} \partial_{1}\right)+v
$$

for some (depending on $k$ ) $\pi^{r}$-vertical vector $v$ over $j_{0}^{r} \partial_{1}$.

Since $j_{0}^{r} \partial_{1}=j_{0}^{r}\left(\partial_{1}+\frac{1}{(r+1) !}\left(x^{1}\right)^{r+1} \partial_{1}\right)$, there exists a local diffeomorphism $\varphi$ with $j_{0}^{r+1} \varphi=$ id sending the germ at 0 of $\partial_{1}$ into the germ at 0 of $\partial_{1}+$ $\frac{1}{(r+1) !}\left(x^{1}\right)^{r+1} \partial_{1}$. Such $\varphi$ preserves $j_{0}^{r} \partial_{1}$ and preserves $j_{0}^{r+1}\left(\frac{1}{k !}\left(x^{1}\right)^{k} \partial_{1}\right)$ if $k \geq 1$. So, if $k \geq 1, \varphi$ preserves the left-hand side of (5.1) because of the order argument. Indeed, by Lemma 42.5 in [4, $A$ is of order $\leq r+1$ because $J^{r} T$ is of order $\leq r+1$. Moreover, $\varphi$ preserves $v$. Indeed, the vertical bundle $V J^{r} T$ of $J^{r} T$ is of order $r+1$ because $J^{r} T$ is of order $r+1$.

On the other hand, $\varphi$ does not preserve $\mathcal{J}^{r} \partial_{1}^{C}\left(j_{0}^{r} \partial_{1}\right)$, because

$$
\mathcal{J}^{r}\left(\frac{1}{(r+1) !}\left(x^{1}\right)^{r+1} \partial_{1}\right)^{C}\left(j_{0}^{r} \partial_{1}\right)=j_{0}^{r}\left(\frac{1}{r !}\left(x^{1}\right)^{r} \partial_{1}\right) \neq 0
$$


where we identify $E_{x}$ with $V_{v} E$ in the obvious way, for any vector bundle $E \rightarrow M$, $v \in E_{x}$, and $x \in M$. Indeed, if $\varphi_{t}$ is the flow of $\frac{1}{(r+1) !}\left(x^{1}\right)^{r+1} \partial_{1}$, then

$$
\begin{aligned}
& \mathcal{J}^{r}\left(\frac{1}{(r+1) !}\left(x^{1}\right)^{r+1} \partial_{1}\right)^{C}\left(j_{0}^{r} \partial_{1}\right)=\frac{d}{d t}{ }_{\mid t=0} J^{r} T \varphi_{t}\left(j_{0}^{r} \partial_{1}\right)=\frac{d}{d t}{ }_{\mid t=0} j_{0}^{r}\left(\left(\varphi_{t}\right)_{*} \partial_{1}\right) \\
& \quad=j_{0}^{r}\left(\frac{d}{d t}{ }_{\mid t=0}\left(\varphi_{t}\right)_{*} \partial_{1}\right)=j_{0}^{r}\left(\left[\partial_{1}, \frac{1}{(r+1) !}\left(x^{1}\right)^{r+1} \partial_{1}\right]\right)=j_{0}^{r}\left(\frac{1}{r !}\left(x^{1}\right)^{r} \partial_{1}\right) .
\end{aligned}
$$

Consequently, $\lambda_{k}=0$ for $k \in\{1, \ldots, r\}$, as well. Then $T \pi^{r} \circ A(X)\left(j_{x}^{r} Y\right)=$ $\lambda_{0} X(x)$ for any $X \in \mathcal{X}(M)$ and any $j_{x}^{r} Y \in J^{r} T M$. Then replacing $A(X)$ by $A(X)-\lambda_{0} \mathcal{J}^{r} X^{C}$, we may assume that $A(X)$ is vertical for any $X \in \mathcal{X}(M)$ and any $m$-manifold $M$. Let $p r: V J^{r} T M \rightarrow J^{r} T M$ be the projection given by $\left.\frac{d}{d t}\right|_{t=0}\left(j_{x}^{r} Y+\right.$ $\left.t j_{x}^{r} Y_{1}\right) \mapsto j_{x}^{r} Y_{1}$. Then the composition $p r \circ A: T_{\mid \mathcal{M} f_{m}} \rightsquigarrow\left(J^{r} T, J^{r} T\right)$ is an $\mathcal{M} f_{m^{-}}$ natural linear operator. So, by Theorem 5.1, $p r \circ A$ is a constant multiple of $A^{\langle 0\rangle}$. Then $A(X)$ is a constant multiple of $\mathcal{J}^{r} X^{V}$.

The proof of the theorem is thus complete.

\title{
REFERENCES
}

[1] J. Gancarzewicz, Liftings of functions and vector fields to natural bundles, Dissertationes Math. (Rozprawy Mat.) 212 (1983), 55 pp. MR 0697471

[2] J. Gancarzewicz, Relèvements des champs de vecteurs aux fibrés naturels, C. R. Acad. Sci. Paris Sér. I Math. 296 (1983), no. 1, 59-61. MR 0691028.

[3] I. Kolář, On the natural operators on vector fields, Ann. Global Anal. Geom. 6 (1988), no. 2, 109-117. MR 0982760

[4] I. Kolář, P. W. Michor and J. Slovák, Natural operations in differential geometry, SpringerVerlag, Berlin, 1993. MR 1202431

[5] J. Kurek and W. M. Mikulski, Lifting vector fields to the $r$ th order frame bundle, Colloq. Math. 111 (2008), no. 1, 51-58. MR 2353930

[6] M. Kureš and W. M. Mikulski, Natural operators lifting vector fields to bundles of Weil contact elements, Czechoslovak Math. J. 54(129) (2004), no. 4, 855-867. MR 2099999

[7] W. M. Mikulski, Some natural operations on vector fields, Rend. Mat. Appl. (7) 12 (1992), no. 3, 783-803. MR 1205977 .

[8] A. Morimoto, Prolongation of connections to bundles of infinitely near points, J. Differential Geom. 11 (1976), no. 4, 479-498. MR 0445422

[9] K. Yano and S. Ishihara, Tangent and cotangent bundles: differential geometry, Marcel Dekker, New York, 1973. MR 0350650

\author{
Jan Kurek \\ Institute of Mathematics UMCS, M. Curie-Sklodowskiej 1, 20-031 Lublin, Poland \\ kurek@hektor.umcs.lublin.pl
}

Wtodzimierz M. Mikulski $i^{凶}$

Faculty of Mathematics and Computer Science UJ, ul. Łojasiewicza 6, 30-348 Krakow, Poland Wlodzimierz.Mikulski@im.uj.edu.pl

Received: January 25, 2019

Accepted: May 7, 2019 